\title{
Architecture of the Mandibular Condylar Cartilage of Elderly Individuals: A Semiquantitative Light Microscopic Histological Study
}

\author{
German O Ramirez-Yanez ${ }^{1}$, James E Scott ${ }^{2}$
}

\begin{abstract}
Aim: This study aimed to categorize the constituent tissues of the mandibular condylar cartilage of elderly individuals.

Materials and methods: Thirty-three mandibular condyles were collected from 20 human cadavers of individuals between 40 years and 103 years old. Samples were stained with Masson's trichrome and Herovici's stains and, examined under a light microscope.

Results: All samples showed tissues that were categorized as fibrous and hyaline cartilage in the mandibular condylar cartilage. A thick fibrous cartilage layer was differentiated on the top of a thinner hyaline cartilage in all of the examined samples. Undifferentiated cells, as well as mature and hypertrophic chondroblasts, were observed in the layer identified as hyaline cartilage, even though they were not in an organized manner. Conclusion: The observations from this study confirm that both fibrocartilage and hyaline cartilage are still present in the mandibular condylar cartilage of elderly individuals.

Clinical significance: The results from this study infer that the mandibular condylar cartilage could be still able to respond to stimulus in adults. In that context, the results of the present study set the basis for future studies aiming to elucidate the biological activity and the remodeling potential of the tissues at the mandibular condyle in adults.
\end{abstract}

Keywords: Endochondral ossification, Fibrocartilage, Hyaline cartilage, Mandibular condyle.

The Journal of Contemporary Dental Practice (2019): 10.5005/jp-journals-10024-2594

\section{INTRODUCTION}

The mandibular condylar cartilage is composed of two different types of cartilage, a fibrous cartilage and a hyaline cartilage, which interact to maintain the integrity of the tissue. ${ }^{1,2}$ It is considered an important regional adaptive growth center of the mandible, and it plays a key role in the adaptive responses and metabolic capacity of the mandibular condyle. ${ }^{3}$

The superficial fibrous cartilage, known as fibrocartilage, contains elongated cells, fibroblasts, and numerous fibers of collagen type I. Its function is to dissipate the compressive loads delivered on the cartilage and bone during the dynamics of the temporomandibular joint (TMJ). ${ }^{4,5}$ Immediately underneath is the hyaline cartilage. It contains more rounded cells embedded in a more or less homogeneous extracellular matrix mainly composed of collagen type II. The hyaline cartilage maintains the growth and adaptive features of the mandibular condylar cartilage through endochondral ossification. ${ }^{2,6}$

Longitudinal growth of the mandible occurs at the highest rate during the first 5 years of life, ${ }^{7}$ followed by a plateau, and then a new growth spurt during puberty. ${ }^{3}$ Over that time, the mandibular condylar cartilage plays an important role in the longitudinal increase of the mandible, ${ }^{1,2}$ undergoing morphological age-related alterations. There is a general consensus that the capacity of the hyaline cartilage to undergo endochondral ossification is not maintained after the third decade of life in humans, as it becomes replaced by fibrocartilage..$^{8,9}$ However, Luder observed remodeling activity in the adult condyle with a transient intensification of endochondral ossification around the fourth and fifth decades of life in humans. ${ }^{10,11}$ Those observations suggest that the hyaline cartilage in the mandibular condyle may still produce some endochondral
${ }^{1}$ Aurora Kids Dentistry, Aurora, Canada

${ }^{2}$ Department of Oral Biology, College of Dentistry, University of Manitoba, Winnipeg, Canada

Corresponding Author: German O Ramirez-Yanez, Aurora Kids Dentistry, Aurora, Canada, Phone: +1 647963 5716, e-mail: ramirezger@gmail.com How to cite this article: Ramirez-Yanez GO, Scott JE. Architecture of the Mandibular Condylar Cartilage of Elderly Individuals: A Semiquantitative Light Microscopic Histological Study. J Contemp Dent Pract 2019;20(7): 768-772.

Source of support: Nil

Conflict of interest: None

ossification, and so, some remodeling activity may remain during the adulthood at the mandibular condylar cartilage. Such a statement may be supported by a cephalometric study reporting changes in the dimensions of the mandible in adults, ${ }^{12}$ suggesting that some late growth may occur in the mandible at adulthood, which may occur at the mandibular condylar cartilage. To elucidate that dilemma, studies are required to identify markers of cartilage matrix deposition and bone mineralization at the mandibular condylar cartilage of adults.

Before biological studies are developed to elucidate the biology of the adult mandibular condylar cartilage, it is necessary to identify the architectural features of that cartilage. In that way, it will be feasible to establish the basis for future studies intending to clarify if the mandibular condylar cartilage may participate in the mandibular remodeling activity in humans at later ages. Therefore, this histological study aimed to describe the architectural composition of the mandibular condylar cartilage of adults and elders, as well as to discuss the morphological changes that may occur in that cartilage with aging. 

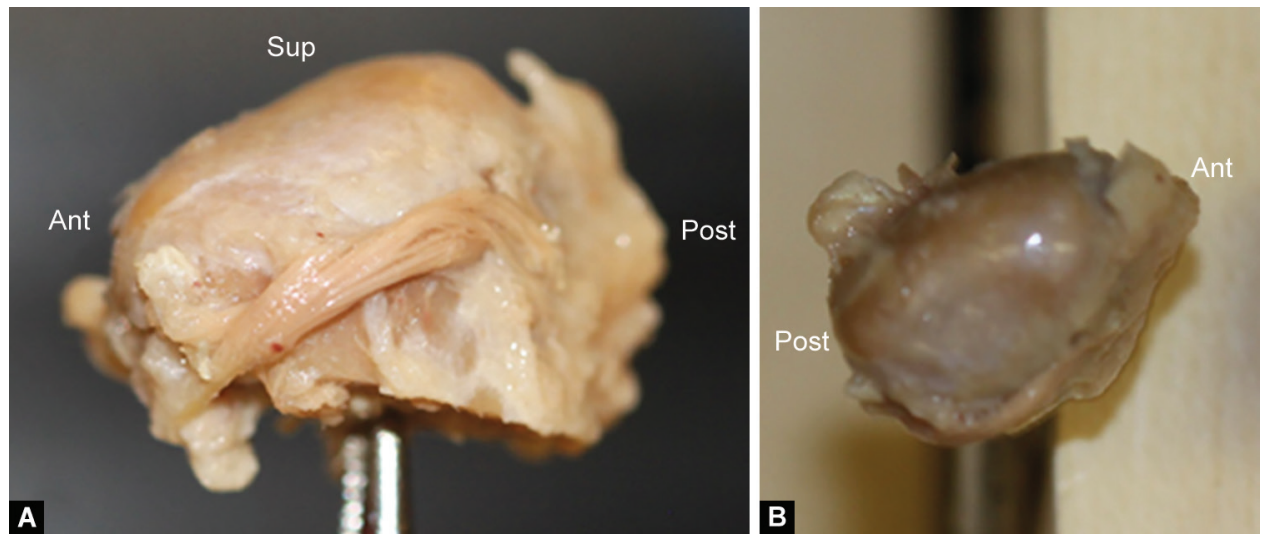

Figs $1 \mathrm{~A}$ and B: The heads of the mandibular condyle harvested from two subjects included in the study. They show the anterior and posterior areas of the mandibular condyle, as well as the superior area in sample $\mathrm{A}$

\section{Materials and Methods}

Over a 2-year period, 33 human mandibular condyles were collected from 20 cadavers at the Anatomy Gross Laboratory, College of Dentistry, University of Manitoba. Ethics approval was granted for the collection of the samples (Ref. no. H2012:154). Data collected for each cadaver included age, gender, and presence or absence of teeth. Unilateral condyle samples were collected from seven cadavers because the contralateral condyles were missing or damaged from previous dissections. Therefore, the 33 mandibular condyles harvested for this study came from a total of 20 human cadavers.

The cadavers were preserved with a mixture of 50/50 methanol/ ethylene glycol/phenol. The fixative was allowed to infiltrate for a minimum of 6 months at $8^{\circ} \mathrm{C}$, prior to dissection. Soft-tissue dissection was completed by removing the muscles surrounding the TMJ. The head of the mandibular condyle was removed using a bone saw and manually de-fleshed (Fig. 1). All samples were further fixed in paraformaldehyde over a 72-hour period.

All samples were processed at the Oral Pathology Laboratory of the College of Dentistry, University of Manitoba, Winnipeg, Canada. Samples were decalcified with $5 \%$ formic acid for 30 days and rinsed with distilled water for 2 days. The condyles were tested for complete decalcification with $1 \mathrm{~mL}$ of saturated ammonium oxalate and $5 \mathrm{~mL}$ of $5 \%$ formic acid. Any sign of precipitate would translate to the presence of calcium. After confirming decalcification radiographically, the samples were stored in a phosphate buffer solution until processed for histology.

Tissues were embedded in paraffin using a paraffin embedding station (Leica Biosystems, Nussloch, Germany), and then processed using the Shandon Citadel 1000 Tissue Processor (Shandon Scientific Limited, Cheshire, England). Samples were then sagittally sectioned (thickness $5 \mu \mathrm{m}$ ) (Reichert Jung 820 II Histocut Microtome, Nussloch, Germany). Ten slides were obtained from each sample, and so, 330 slides were obtained for this study. Then, five slides from each of the samples were stained with Herovici's stain and the remaining five slides from each of the samples were stained with Masson's trichrome. The staining techniques are described later.

The anterior zone of the mandibular condyle is a load-bearing zone, which is highly affected by the dynamics of the TMJ. ${ }^{13}$ Conversely, the posterior zone is a non-load-bearing area, which has been reported to be the area where mandibular growth occurs through endochondral ossification. ${ }^{2,13}$ In this descriptive report, the area of interest was the non-load-bearing posterior zone of the mandibular condylar cartilage (Fig. 2).

\section{Herovici's Staining}

Herovici's staining is a polychrome stain originally proposed for differentiating precollagen (blue) from collagen (red) $)^{14}$ and involves several solutions. The first solution (A) was prepared with $0.25 \mathrm{~g}$ of celestine blue plus $2.5 \mathrm{~g}$ iron alum dissolved in $50 \mathrm{~mL}$ of distilled water. The solution was heated and boiled for 3-5 minutes, filtered, and $10 \mathrm{~mL}$ of glycerol was added. Solution $B$ was made by adding $50 \mathrm{~mL}$ of $1 \%$ alcoholic hematoxylin to $100 \mathrm{~mL}$ of $5.0 \%$ aqueous aluminum sulfate, then boiling. After cooling, $100 \mathrm{~mL}$ of distilled water, $10 \mathrm{~mL}$ of $4 \%$ aqueous $\mathrm{FeCl}_{3}$, and $1 \mathrm{~mL}$ of $\mathrm{HCl}$ were added. Solution C was made by diluting $0.25 \mathrm{~g}$ of metanil yellow in $60 \mathrm{~mL}$ of distilled water containing five drops of glacial acetic acid. Solution $D$ was a mixture of $60 \mathrm{~mL}$ distilled water and five drops of glacial acetic acid. Solution $\mathrm{E}$ contained $60 \mathrm{~mL}$ of distilled water and two drops of $\mathrm{Li}_{2} \mathrm{CO}_{3}$. Solution $\mathrm{F}$ was made by mixing two solutions, the first one containing $0.05 \mathrm{~g}$ of methyl blue in $50 \mathrm{~mL}$ of distilled water, and the second solution containing $0.1 \mathrm{~g}$ of acid fuchsine in $50 \mathrm{~mL}$ of saturated aqueous picric acid. Then, that mixture was added with $10 \mathrm{~mL}$ of glycerol and $0.5 \mathrm{~mL}$ of saturated aqueous $\mathrm{Li}_{2} \mathrm{CO}_{3}$.

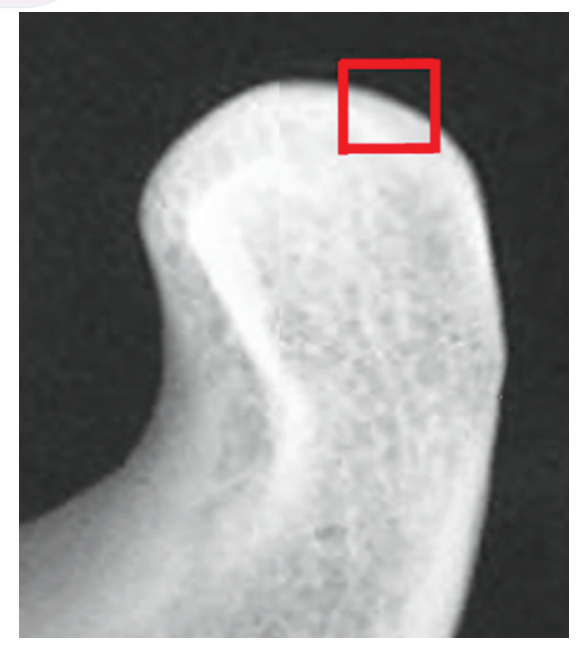

Fig. 2: Radiograph of one of the studied condyles showing the superoposterior area of the mandibular condyle observed in the stained slides used in the present study 
Samples were stained with solution A for 5 minutes followed by a wash with running water for 1-2 minutes. Next, samples were placed in solution $B$ for 5 minutes, washed in running water for 15-30 minutes, and then stained with solution $C$ for 2 minutes. After solution C, samples were immersed in solution D for a couple of minutes, rinsed with water, and then placed in solution $E$ for 2 minutes followed by flooding the slides with solution $F$ for 2 minutes. Finally, the tissues were placed in a solution of $1 \%$ acetic acid for 2 minutes, dehydrated, cleared, and cover-slipped.

\section{Masson's Trichrome Staining}

Masson's trichrome is a tricolor staining protocol used in histology to identify collagen tissues, such as muscle and bone, as well as the presence of cells in those tissues. For that purpose, three solutions were previously prepared. The Biebrich scarlet-acid fuchsin solution was made of $90.0 \mathrm{~mL}$ of $1 \%$ aqueous Biebrich scarlet, $10.0 \mathrm{~mL}$ of $1 \%$ aqueous acid fuchsin, and $1.0 \mathrm{~mL}$ of glacial acetic acid. The phosphomolybdic-phosphotungstic acid solution was made of $5.0 \mathrm{~g}$ of phosphomolybdic acid, $5.0 \mathrm{~g}$ of phosphotungstic acid, and $200.0 \mathrm{~mL}$ of distilled water. The aniline blue solution was made from $2.5 \mathrm{~g}$ of aniline blue, $2.0 \mathrm{~mL}$ of acetic acid, and $100.0 \mathrm{~mL}$ of distilled water. The light green solution was made of $5.0 \mathrm{~g}$ of light green dye, $250.0 \mathrm{~mL}$ distilled water, and $2.0 \mathrm{~mL}$ glacial acetic acid.

Tissues were initially immersed in Bouin's solution for 1 hour at $56^{\circ} \mathrm{C}$. This solution consisted of $75.0 \mathrm{~mL}$ of picric acid-saturated aqueous solution, $25.0 \mathrm{~mL}$ of $37-40 \%$ formalin, and $5.0 \mathrm{~mL}$ of glacial acetic acid. After cooling and washing the tissues in running water, samples were rinsed again in distilled water and immersed in the Biebrich scarlet-acid fuchsin solution for 15 minutes. After another rinse in distilled water, tissues were exposed to phosphomolybdic acid-phosphotungstic acid solution for 10-15 minutes before adding an aniline blue solution. Tissues were then immersed in 5\% aqueous phosphotungstic acid for 15 minutes before adding the light green counterstain. After submerging tissues in aniline blue solution for 5-10 minutes, samples were rinsed in distilled water and then in 1\% acetic water for 3-5 minutes. Next, the samples were passed twice through $95 \%$ absolute alcohol, and then xylene. Finally, samples were mounted in Permount.

\section{Data Collection and Evaluation}

Sagittal sections in an anteroposterior perspective were examined under a light microscope at $10 \times$ magnification. As mentioned above, the supero-posterior area of the surface of the mandibular condyle was the area of observation in this study. All the stained slides were observed by one of the investigators (GRY) who had the histological knowledge on how to identify the various layers and cells contained in the mandibular condylar cartilage. ${ }^{15,16}$

\section{Results}

The ages of the deceased patients (i.e., cadavers) ranged from 47 to 103 years, with a mean age of 80.95 years. Eleven of the 20 subjects involved in the study (55.0\%) were edentulous. Seventeen of the 20 subjects (85.0\%) were females. The demographics for the subjects and samples are presented in Table 1.

On the one hand, Herovici's stain produced the following results: nuclei in the cells appeared black, the cytoplasm did not stain, the fibers containing collagen I in the fibrocartilage stained light blue, the matrix in the hyaline cartilage appeared blue, and the mineralized tissue stained red. Hematopoietic tissue stained purple (Fig. 3). On the other hand, Masson's trichrome stain produced the
Table 1: Descriptive demographics of the 33 human mandibular condyles from 20 cadavers collected for the study

\begin{tabular}{lcc}
\hline Category & Sample \# & Percent (\%) \\
\hline Gender & & \\
Male & 3 & 15.0 \\
Female & 17 & 85.0 \\
Age & & \\
$40-60$ & 2 & 10.0 \\
$60-80$ & 6 & 30.0 \\
$80+$ & 12 & 60.0 \\
Edentate & & \\
Yes & 11 & 55.0 \\
No & 9 & 45.0 \\
\hline
\end{tabular}

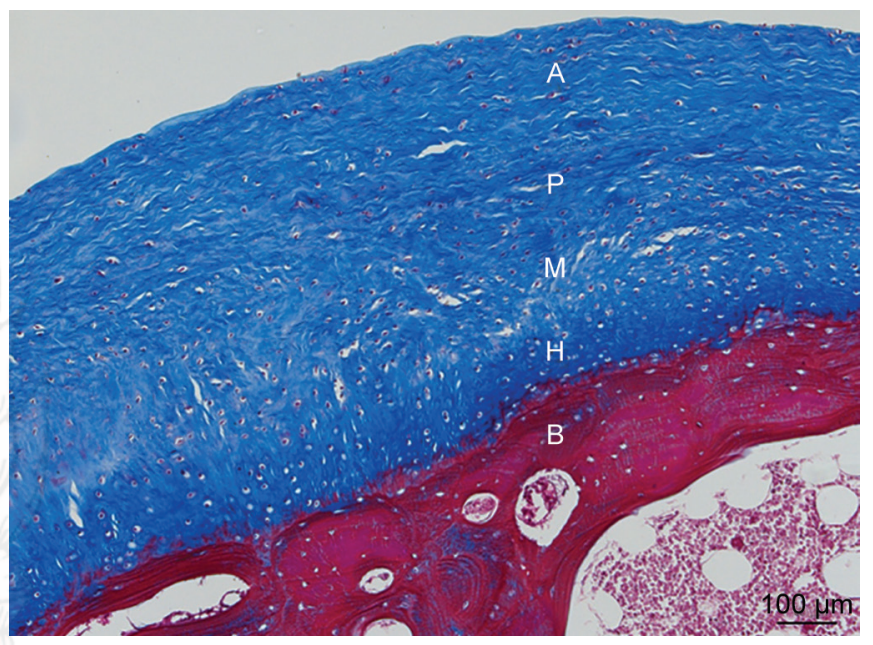

Fig. 3: Mandibular condylar cartilage from a 71-year-old human stained with Herovici's stain. It shows four different layers composing the mandibular condylar cartilage: the thick layer on the surface categorized as fibrocartilage ( $F$ ) covering three deeper layers; a layer containing small cells (P: proliferative) followed by one layer containing rounded cells (M: mature); and finally, a layer containing bigger and elongated cells ( $\mathrm{H}$ : hypertrophic). Notice a thin pink area underneath the hypertrophic layer and the mineralized bone (B), which may suggest an area of endochondral ossification. $10 \times$ magnification

following results: nuclei in the cells appeared black, the cytoplasm did not stain, fibers containing collagen I in the fibrocartilage stained blue, the matrix in the hyaline cartilage appeared pink/ light purple, and mineralized tissue stained purple. Hematopoietic tissue stained blue/gray (Fig. 4).

Under light microscopy, four histological layers were categorized in the mandibular condylar cartilage of all samples (Figs 3 and 4). The upper layer appeared as a dense, regular connective tissue with parallel fibers running at the articular surface, containing fibrocartilage, and fibroblasts. This was the thicker layer composing the mandibular condylar cartilage. Immediately under that upper layer, there was a thin layer containing a higher number of small rounded or small elongated cells, which was categorized as the proliferative layer. The deepest two layers were composed of mostly rounded cells, with the deeper one containing bigger cells, looking as hypertrophic cells. So, they were categorized as the mature and hypertrophic layers. Beneath the mandibular condylar cartilage, there was trabecular bone containing bone cells and Haversian systems (Figs 3 and 4). 


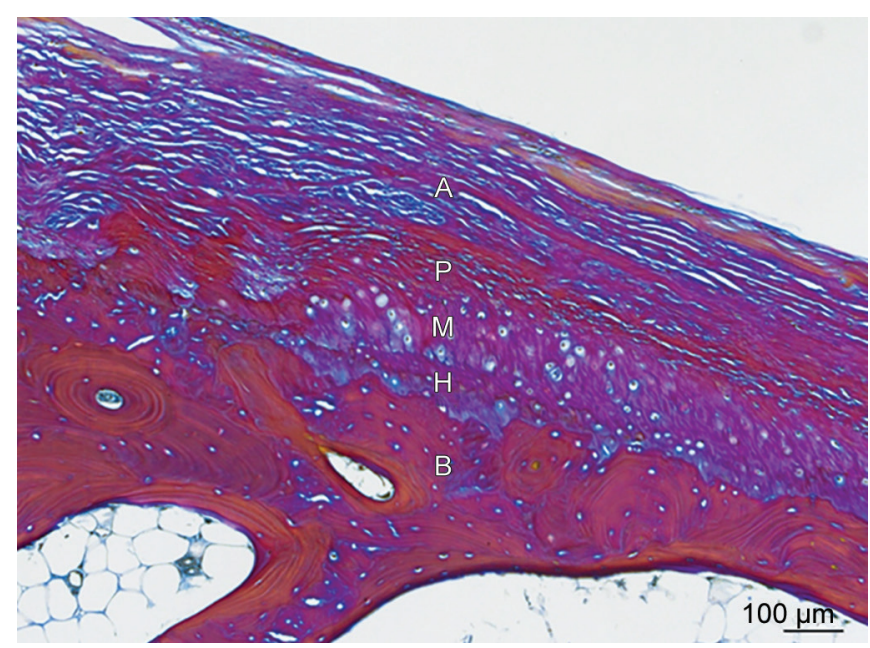

Fig. 4: Mandibular condylar cartilage from a 94-year-old human stained with Masson's trichrome stain, showing the four characteristic layers of the mandibular cartilage (A: articular; P: proliferative; $M$ : mature; $\mathrm{H}$ : hypertrophic), as well as bone (B). Note that the hypertrophic $(\mathrm{H})$ layer shows a light purple coloration comparing with the darkest purple color observed on the bone tissue immediately underneath (B), which suggests the presence of unmineralized cartilage matrix. 10X magnification

All the studied samples showed a mandibular condylar cartilage composed by a layer with a higher contain of fibers at the surface of the mandibular condyle, which covered a thinner layer containing various types of cells, which was identified as a hyaline-like cartilage. Although it was possible to categorize four different layers in the mandibular condylar cartilage, those were not organized in such a way to clearly identify the limits between one layer and the neighbor ones.

Another observation in the samples studied here was a thin zone of early mineralized tissue at the border between the mandibular condylar cartilage and the mineralized bone which was observed on both stains. It appeared as a pink region at the bottom of the hypertrophic layer in Herovici's stain (Fig. 3), and as a light blue region at the bottom of the hypertrophic layer on Masson's trichrome stain (Fig. 4).

The staining protocols used in this study permitted to describe in detail the architectural composition of the mandibular condylar cartilage of adults and elders, which had not been reported before.

\section{Discussion}

The mandibular condylar cartilage plays an important role in the development and function of the TMJ. ${ }^{3}$ Numerous cells have been observed in the layer overlying the calcified cartilage zone in the mandibular condyle of elderly individuals, ${ }^{17}$ but the type of cells has not been properly identified at that age. This study used differential histological stains, which further permit to differentiate various types of cells in the studied tissue, as well as to observe various tissues in the studied area. That would be no possible to observe with a simpler stain, such as H\&E. Thus, the histological observations from this study permitted to identify various layers, containing different cells in the mandibular condylar cartilage at late adulthood and senescence, and, therefore, it may be reported that the mandibular condylar cartilage at adulthood in humans still contains both, the fibrotic and hyaline cartilage layers.

It was also observed that the fibrotic layer contained fibroblasts, whereas the hyaline cartilage contained cells with a characteristic chondroblastic morphology at different developmental stages, as well as, hypertrophic cells. Even though the architecture and characteristics of the cartilage at the mandibular condyle resemble those tissues in younger individuals ${ }^{18,19}$ and animals, ${ }^{15,16}$ this is a descriptive study, which does not permit to establish that those cells and tissues have a similar biological activity as that reported in younger tissues. Therefore, further studies are required to determine the biological activity and response of those tissues and cells contained in the mandibular condylar cartilage of elder humans.

These results concur with those studies suggesting that the morphology of the mandibular condylar cartilage varies in adults, but both fibrous and hyaline cartilages are still present. ${ }^{18,19}$ Additionally, this report permitted to observe constituent cells in both, the fibrocartilage and the hyaline-like cartilage, with the later holding different types of cells at various developmental stages, as reported by Luder and Ishibashi et al. ${ }^{11,18}$ However, the various groups of cells encompassed in the hyaline cartilage were not well organized with clear limits, which is one of the characteristics found in younger human samples or animal studies. ${ }^{15,16,20}$ It may suggest that the biological activity of the tissues in the mandibular condylar cartilage at elderly individuals may be quite different to that reported at an early age.

Conversely, the current results disagree with those stating that in humans, the hyaline cartilage in the mandibular condyle disintegrates or is replaced by bone/fibrocartilage after the third decade of life. ${ }^{8,21}$ The present results showed that a thin layer of hyaline-like cartilage containing chondrocytes at different developmental stages is present until senescence in humans, even though it is significantly reduced in the elderly individuals. Furthermore, the presence of cells in the hyaline-like cartilage may suggest that that cartilage observed in the samples included in this study would still have the potential of endochondral ossification. That suggestion would be supported by the observation of a thin layer of early mineralized tissue identified at the border between the mandibular cartilage and the mineralized bone in all the studied samples, which appears as a grey zone in the tissues stained with Masson's trichrome technique. In that context, this report tends to support Luder's study, ${ }_{1}^{11}$ where it was stated that an intermediate zone in the mandibular condylar cartilage appears to be associated with continued maintenance and adaptive articular remodeling in the adult mandibular condylar cartilage.

It can be said that there are age-related morphological changes in the mandibular condylar cartilage in humans. ${ }^{11}$ Although there could be a reduction of cellularity in the hyaline cartilage of elderly humans, ${ }^{19}$ and the various layers in the hyaline cartilage may appear disorganized, it does not mean that the hyaline cartilage reaches a state of acellularity or disappears, as it has been proposed by other authors. ${ }^{8,21}$ In other words, this report suggests that even though the hyaline cartilage composing the mandibular condylar cartilage may be reduced in thickness and be less organized in the elderly individuals, it may remain viable throughout life in humans and maintain mature and hypertrophic chondroblasts, which are required for life-long tissue maintenance and adaptive remodeling. ${ }^{22}$ Future studies may correlate the structural and cellular changes that may occur at different ages, as this study did not discriminate the age of the deceased patient in correlation to the structural changes.

It is necessary to keep in mind that the morphology of the mandibular condylar cartilage varies depending on the observed zone. The anterior zone of the mandibular condyle is a load-bearing zone, which is highly affected by the dynamics of the TMJ. ${ }^{13}$ On the opposite side, the posterior zone is a non-load-bearing area, which 
has been reported to be the area where mandibular growth occurs through endochondral ossification. ${ }^{2,13}$ In this descriptive report, the observations focused on that posterior area of the mandibular condyle in the studied samples. As it was mentioned before, that nonload-bearing area maintains a fibrotic layer and a zone of hyaline-like cartilage, containing cells at different developmental stages. That may infer that the mandibular condylar cartilage still might play a role in some of the variations occurring in the dimensions of the mandible in the adulthood. It might explain the dimensional changes reported in the mandible of adults in a cephalometric study. ${ }^{12}$ However, further studies are required to elucidate the role of the mandibular condylar cartilage in that phenomenon.

Although the observations from this report permitted to identify a fibrotic and a hyaline-like cartilage in elderly individuals, the thicknesses of the various layers contained in the hyaline cartilage at different ages or the mitotic and biological activities of the cells composing both of those types of cartilages were assessed. The relevance of the results from this study is that they described the particular architecture of the mandibular condylar cartilage in adults, as well as established the morphological differences of that tissue between adults or elders and that in younger subjects, as reported by Luder. ${ }^{11}$ In that context, this study established the basis for future investigations where the differences between the various collagen types composing the superficial and deeper layers in the adult mandibular condylar cartilage, as well as the biological activity of those cells contained in the mandibular condylar cartilage of adults and elders could be identified. In that way, the potential of endochondral ossification that could still occur at the mandibular condylar cartilage in the adulthood might be elucidated and explained.

\section{ConCLUSION}

The observations from this study permitted to identify the architecture of the mandibular condylar cartilage, which contains a zone of fibrotic tissue and a zone of hyaline cartilage covering the mandibular condyle of elderly individuals. Both layers maintain their cellularity, which infers that the mandibular condylar cartilage may maintain a biological activity throughout life in humans. Furthermore, the differences in the mandibular condylar cartilage morphology in elderly subjects with that of younger subjects reported by other authors were established. Thus, this study sets the basis for future investigations aiming to elucidate the biological activity and response of the mandibular condylar cartilage in adults and elders.

\section{Clinical Significance}

The present results are of clinical significance as they infer the mandibular condylar cartilage could be still able to respond to stimulus in adults. In that context, the current results set the basis for future studies intending to understand the biology of the mandibular condylar cartilage in adults and potential treatments that may help patients with degenerative processes at the TMJ, such as osteoarthritis.

\section{References}

1. Petrovic A. Auxologic categorization and chronobiologic specification for the choice of appropriate orthodontic treatment. Am J Orthod Dentofacial Orthop 1994;105(2):192-205. DOI: 10.1016/ S0889-5406(94)70114-8.
2. Petrovic AG. Mechanisms and regulation of mandibular condylar growth. Acta Morphol Neerl Scand 1972;10(1):25-34.

3. Copray J, Dibbets J, et al. The role of condylar cartilage in the development of the temporomandibular joint. Angle Orthod 1988;58(4):369-380.DOI: 10.1043/0003-3219(1988)058<0369:TROCCI>2.0.CO;2.

4. Hansson T, Oberg T, et al. Thickness of the soft tissue layers and the articular disk in the temporomandibular joint. Acta Odontol Scand 1977;35(2):77-83. DOI: 10.3109/00016357709055993.

5. Nanci A. Ten Cate's Histology: Development, structure and function. St. Louis, Missouri: Mosby, 2008.

6. Petrovic AG, Stutzmann JJ, et al. The final length of the mandible: is it genetically predetermined? Craniofacial Biology. Univ. Michigan, 1981; pp. 105-126; Vol Monograph 10.

7. Liu $Y$, Behrents $R$, et al. Mandibular growth, remodeling, and maturation during infancy and early childhood. Angle Orthod 2010;80:97-105. DOI: 10.2319/020309-67.1.

8. Moffett B. The morphogenesis of the temporomadibular joint. Am J Orthod 1966;52:401-415. DOI: 10.1016/0002-9416(66)90120-5.

9. Wright $D$, Moffett $B C$. The postnatal development of the human temporomandibular joint. Am J Anat 1974;141:235-250. DOI: 10.1002/ aja.1001410206.

10. Luder HU. Frequency and distribution of articular tissue features in adult human mandibular condyles: a semiquantitative light microscopic study. Anat Rec 1997;248(1):18-28. DOI: 10.1002/ (SICI)1097-0185(199705)248:1<18::AID-AR3>3.0.CO;2-B.

11. Luder $\mathrm{H}$. Age changes in the articular tissue of human mandibular condyles from adolescence to old age: a semiquantitative light microscopic study. Anat Rec 1998;251(4):439-447. DOI: 10.1002/ (SICI)1097-0185(199808)251:4<439::AID-AR3>3.0.CO;2-N.

12. Pecora $\mathrm{N}$, Baccetti $\mathrm{T}$, et al. The aging craniofacial complex: a longitudinal cephalometric study from late adolescence to late adulthood. Am J Orthod Dentofacial Orthop 2008;134:496-505. DOI: 10.1016/j.ajodo.2006.11.022.

13. Thilander B, Carlsson G, et al. Postnatal development of the human temporomandibular joint. I. A histological study. Acta Odontol Scand 1976;34(2):117-126. DOI: 10.3109/00016357609026564.

14. Herovici C. A polychrome stain for differentiating precollagen from collagen. Stain Technol 1963;38:204-205.

15. Ramirez-Yanez G, Daley $T$, et al. Incisor disocclusion in rats affects mandibular condylar cartilage at the cellular level. Arch Oral Biol 2004;49(5):393-400. DOI: 10.1016/j.archoralbio.2003.11.005.

16. Ramirez-Yañez $G$, Young $W$, et al. Influence of growth hormone on the mandibular condylar cartilage of rats. Arch Oral Biol 2004;49(7):585590. DOI: 10.1016/j.archoralbio.2004.02.004.

17. Flygare $L$, Klinge $B$, et al. Calcified cartilage zone and its dimensional relationship to the articular cartilage in the human temporomandibular joint of elderly individuals. Acta Odontol Scand 1993;51:183-191. DOI: 10.3109/00016359309041164.

18. Ishibashi H, Takenoshita $\mathrm{Y}$, et al. Age-related changes in the human mandibular condyle: a morphologic, radiologic, and histologic study.J Oral Maxillofac Surg 1995;53:1016-1023. DOI: 10.1016/0278-2391(95)90117-5.

19. Oberg T, Carlsson G. Macroscopic and microscopic anatomy of the temporomandibular joint. In: Zarb G, Carlsson G ed., Temporomandibular joint function and dysfunction. Copenhagen: Munksgaard; 1979. pp. 101-118.

20. Petrovic A, Stutzmann J, et al. Control processes in postnatal growth of condylar cartilage of the mandible. In: McNamara JAJ ed., Determinants of mandibular form and growth. Monograph, 4th ed. Ann Arbor: University of Michigan; 1975. pp. 14-57.

21. Katakami K, Shimoda S, et al. Histological investigation of osseous changes of mandibular condyles with backscattered electron images. Dentomaxillofac Radiol 2008;37:330-339. DOl: 10.1259/dmfr/93169617.

22. Hattori K, Takakura Y, et al. Which cartilage is regenerated, hyaline cartilage or fibrocartilage? Non-invasive ultrasonic evaluation of tissue-engineered cartilage. Rheumatology 2004;43:1106-1108. DOI: 10.1093/rheumatology/keh256. 